\title{
Recorrido geológico y minero por las comarcas del Alt Maestrat del Maestrazgo y de Gúdar Javalambre: desde la Pobla de Sant Miquel al Santuari de I’Estrella y a Mosqueruela
}

Josep Maria Mata-Perelló

Joaquim Sanz Balagué

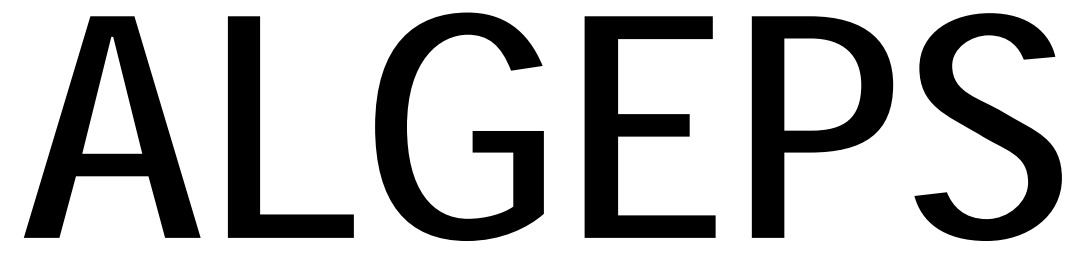

\section{REVISTA DE GEOLOGIA}

\section{n. 3}

JULIOL 2015 


\title{
RECORRIDO GEOLÓGICO Y MINERO POR LAS COMARCAS DEL ALT MAESTRAT DEL MAESTRAZGO Y DE GÚDAR JAVALAMBRE: DESDE LA POBLA DE SANT MIQUEL AL SANTUARI DE L'ESTRELLA Y A MOSQUERUELA
}

\author{
Josep Maria Mata-Perelló \\ Museu de geologia Valentí Masachs, Escola Politècnica Superior d'Enginyeria de Manresa \\ (EPSEM), Universitat Politècnica de Catalunya · BarcelonaTech (UPC), 08272 Manresa, Spain
}

\section{Joaquim Sanz Balagué}

Departament d'Enginyeria Minera i Recursos Naturals (EMRN), Escola Politècnica Superior d'Enginyeria de Manresa (EPSEM), Universitat Politècnica de Catalunya - BarcelonaTech (UPC), 08272 Manresa, Spain

Paraules clau: Patrimoni geològic i miner; Sistema ibèric; Aragó

\begin{abstract}
Resumen
Itinerario realitzado el dia 6 de Diciembre de 2014. En este caso, el presente itinerario se desarrollará totalmente por la denominada Rama Aragonesa de la Cordillera Ibérica. En este recorrido se irán encontrando afloramientos de los materiales mesozoicos (del Triásico, del Jurásico y del Cretácico, especialmente de este último). En concreto, el recorrido se realizará en buena parte por la denominada Cuenca del Maestrazgo. Los materiales antes mencionados se hallan levemente influenciados por las características la denominada Zona de Enlace de la Cordillera Ibérica con el Sistema Mediterráneo; aunque van a predominar las estructuras ibéricas (WNW-ESE) sobre las "catalanas" (NNE-SSW), al hallarnos plenamente dentro del dominio ibérico.

Así, el recorrido del itinerario transitará, en buena parte, por la gran comarca histórica MaestratMaestrazgo, (coincidiendo en parte con la unidad geológica del mismo nombre). Aun así, el recorrido se efectuará por dos comarcas: por la del Alt Maestrat (de las tierras de Castellón) y por la de Gúdar - Javalambre (esta última de Teruel). Asimismo, se realizaran hijuelas por la comarca del Maestrazgo. Sin embargo, la totalidad del recorrido, como hemos dicho se sitúa dentro de la Cuenca del Maestrazgo y dentro del Maestrazgo Histórico.
\end{abstract}




\section{Objectivos fundamentales}

Los objetivos fundamentales que se pretenden conseguir con la realización de este itinerario, se pueden concretar en los siguientes aspectos generales:

1. Observación de los materiales mesozoicos (que según los lugares se reparten entre el Triásico, el Jurásico y el Cretácico, con un claro predominio de este último) que encontraremos por diferentes lugares del recorrido. Estos materiales forman parte del Sistema Ibérico en la Cuenca del Maestrazgo.

2. Observación de la estructura, en este recorrido, del borde oriental de la denominada Cuenca del Maestrazgo. Estas estructuras se hallan también influenciadas por la Zona de Enlace de la Cordillera Ibérica con el Sistema Mediterráneo.

3. Observación de las diferentes mineralizaciones que iremos encontrando a lo largo del recorrido.

4. Observación de las explotaciones mineras desarrolladas sobre las mineralizaciones anteriores, y también sobre otras, a lo largo del recorrido del itinerario.

5. Observación de los impactos producidos sobre el Medio Natural, como consecuencia de las explotaciones anteriores y (si se da el caso) de las restauraciones llevadas a cabo, con la finalidad de mitigar los impactos anteriores.

6. Observación, a lo largo de todo el recorrido, de los distintos LIG (Lugares de Interés Geológico) i de los distintos LIPM (Lugares de Interés del Patrimonio Minero), integrantes de nuestro Patrimonio Geológico y Minero. Entre los primeros, cabe destacar el inmenso Poljé De Mosqueruela. Entre los segundos, no destacaremos ninguno.

\section{Antecedentes}

No tenemos conocimiento de ningún itinerario geológico y minero que discurra por estos sectores, salvo los desarrollados por nosotros. Así, mencionaremos los siguientes: MATAPERELLÓ y MONTANÉ GARCÍA (2004a y 2004b) y finalmente: MATA-PERELLÓ i VILALTELLA (2005 y 2009). Este último, en parte, sigue un recorrido similar al que ahora presentamos.

Por lo que concierne al conocimiento de la estructura geológica de la zona por la que discurre el recorrido del itinerario, nos referiremos a dos trabajos sensiblemente importantes: GUIMERÀ et altri (1992) y RIBA et altri (1976). Igualmente nos referiremos a diversos trabajos del IGME (1986), relativos a los lugares por los que transitará el recorrido. En relación con las características geológicas también citaremos el trabajo de PEÑA et altri (1984). Asimismo, en relación al patrimonio geológico citaremos el trabajo de BURILLO (1996), así como el publicado por la GADMA (2001), dedicado al conjunto de Aragón.

$\mathrm{Y}$, finalmente, por lo que concierne a la descripción de las mineralizaciones, nos referiremos a algunos antecedentes nuestros; concretamente a los trabajos: MATA-PERELLÓ (1991, 1992a y 1992b). 
Todos estos trabajos se hallaran debidamente relacionados en el apartado de REFERENCIAS BIBLIOGRÁFICAS, que figura al final del presente trabajo.

\section{Recorrido del itinerario}

El recorrido del itinerario transitará por dos comarcas, la del Alt Maestrat y la de Gúdar Javalambre (siempre dentro del denominado Maestrazgo Histórico). Así, el trayecto se iniciará en la localidad castellonense de la Pobla de Sant Miquel (situado en la comarca del Alt Maestrat y en el municipio de Vilafranca del Cid). Este lugar se halla en el límite de las comarcas del Alt Maestrat y del Maestrazgo, justo en el límite de las provincias de Castellón y de Teruel.

Desde ahí, se circulara hacía el poniente, por la carretera autonómica valenciana CV - 173. Luego, al entrar en la comarca de Gúdar - Javalambre, esta carretera pasará ser la A - 1701. Así, en este recorrido hacia el poniente, se realizará una hijuela por un camino de tierra, para ir al Santuario de la Virgen de la Estrella. En este tramo del recorrido, se irán efectuando paradas.

Luego, tras retornar a la carretera, el contenido continuará hacia la población de y Mosqueruela (en este último caso entrando en la comarca de Gúdar - Javalambre), con la finalidad de realizar diversas paradas.

\section{Advertencias previas}

Al igual que en otras ocasiones, en los recorridos de RECERCA GEOLÒGICA I NATURALIISTICA..., si se dispone del tiempo suficiente, podrán realizarse todas las paradas e hijuelas propuestas. En caso contrario, recomendamos prescindir de las calificadas con el nombre de PARADAS - CONDICIONALES.

Así se presentará un recorrido aproximado, por diferentes lugares del Maestrazgo Histórico, con una serie de PARADAS situadas entre las poblaciones de la Pobla de Sant Miquel (de Vilafranca del Cid) y Mosqueruela, yendo el recorrido de la comarca actual del Alt Maestrat a la del Gúdar Javalambre, realizando hijuelas por la comarca aragonesa del Maestrazgo, discurriendo por parte de las provincias de Castellón y de Teruel. El recorrido se efectuará por carretera; aunque en algunos tramos se hará por caminos en buen estado de conservación.

En cualquier caso, y como es natural, es necesario tener un absoluto cuidado y respeto hacia el Medio Natural, a lo largo de todo el recorrido. 


\section{Descripción del itinerario}

Como de costumbre, estructuraremos el recorrido del itinerario en una serie de PARADAS, que a continuación iremos viendo. En cada una de estas paradas haremos un breve comentario (geológico o mineralógico, según acontezca). Por otra parte, en cada caso indicaremos (entre paréntesis) la hoja topográfica en donde se halla la parada.

Para ello, utilizaremos solo una hoja del Instituto Geográfico y Catastral de España, publicadas a la escala de 1:50.000. Concretamente la siguiente: 569 (o de Vilafranca del Cid, llamada a veces de Mosqueruela).

Así pues, la relación de las paradas que componen el recorrido de este itinerario, son las siguientes:

\subsection{Parada 1. EL PUENTE DE SAN MIQUEL DEL BALLESTAR, (términos municipales de Vilafranca del Cid, comarca del Alt Maestrat y de la Iglesuela del Cid, comarca del Maestrazgo). (Hoja 569).}

El recorrido del itinerario, lo iniciaremos en este lugar, cerca del puente medieval de Sant Miquel de Ballestar, situado en la comarca del Alt Maestrat y en el municipio de Vilafranca del Cid. En torno a este lugar efectuaremos una nueva parada. Ésta la realizaremos en las inmediaciones del puente sobre el río de las Truchas.

Este lugar se halla situado dentro de la denominada Cuenca del Maestrazgo, en la Rama Aragonesa del Sistema Ibérico, por donde estaremos a lo largo de todo el recorrido del itinerario. Por otra parte, en este lugar afloran los materiales cretácicos. Así, vemos niveles de calcolutitas, calizas y calizas arenosas. Estos materiales se sitúan en el tránsito del Aptiense al Albiense; aunque los niveles superiores ya son del Cenomaniense. Sin embargo, por los alrededores, afloran los niveles arcillosos y arenosos pertenecientes al Albiense

Por otra parte, a poca distancia de aquí (apenas a $1 \mathrm{Km}$, en el municipio turolense de la Iglesuela del Cid), estos materiales han sido explotados para la obtención de losas y losetas para la construcción, concretamente las calizas arenosas. Asimismo, junto al río, en el municipio de la Iglesuela del Cid, hay una explotación de las terrazas del río de las Truchas, que discurre junto al lugar en donde estamos.

Finalmente, cabe decir que en este lugar se halla un majestuoso puente medieval, construido (en buena parte) con losetas de las que afloran por los alrededores. (fotografia 1). 


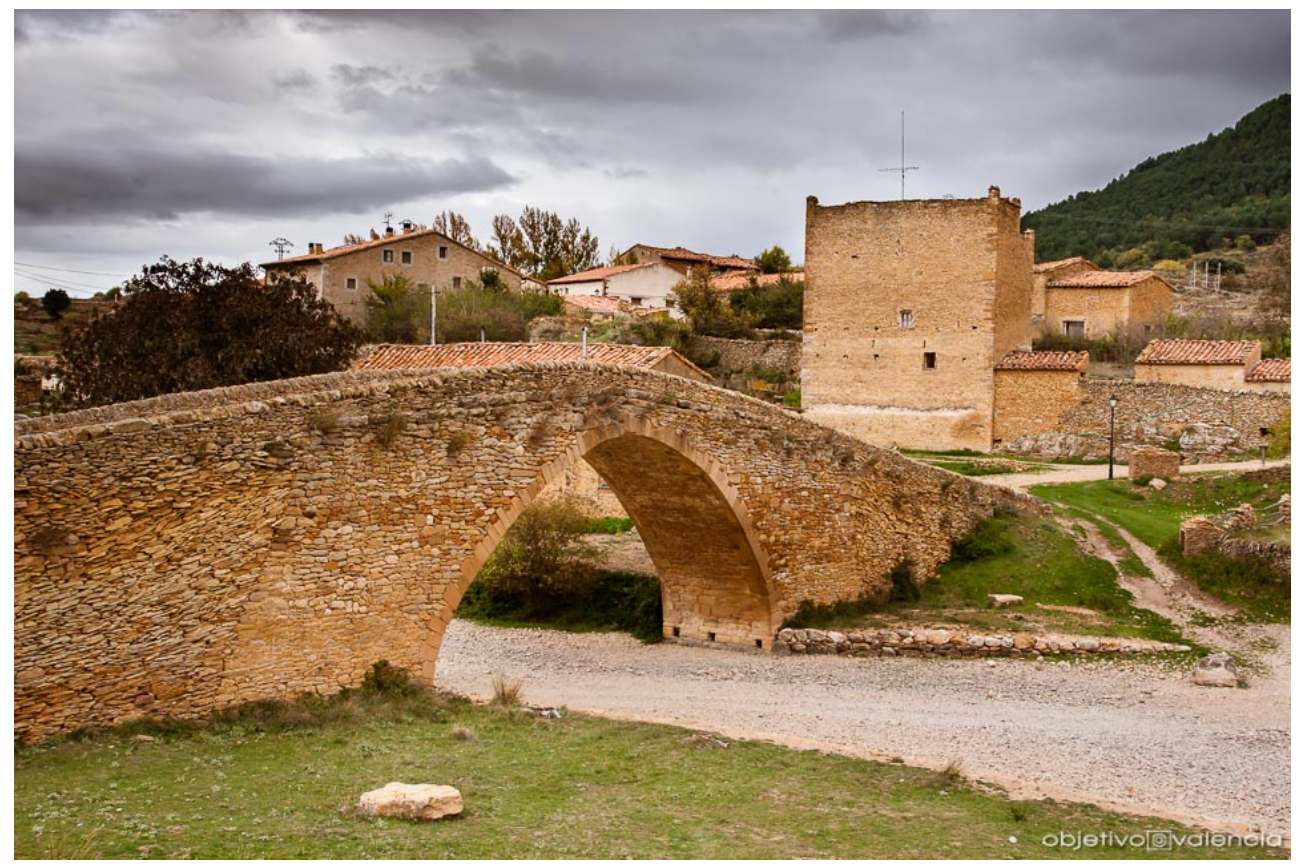

Fotografía 1. El Puente Medieval de Sant Miquel del Ballestar. Vilafranca del Cid Fuente imagen: http://www.espores.org/oci-verd/curs-fotografia-paisatge-valenci\%C3\%A0.html

\subsection{Parada 2. GRAVERA DEL ARROYO DE LAS TRUCHAS, (término municipal de la Iglesuela del Cid, comarca del Maestrazgo). (Hoja 569).}

Después de realizar la parada anterior, cabe la posibilidad de remontar ligeramente el Arroyo de las Truchas, con la finalidad de efectuar una nueva parada. Así, desde la anterior, habremos recorrido algo más de $1 \mathrm{Km}$.

En este recorrido habremos vuelto a encontrar los materiales mesozoicos de los que ya hemos hablado anteriormente.

En este lugar hay una gravera. En ella se aprovechan los materiales detríticos transportados por el Arroyo de las Truchas. Este arroyo tiene una carga eminentemente carbonatada y procede de las inmediaciones de Mosqueruela. (fotografía 2). 


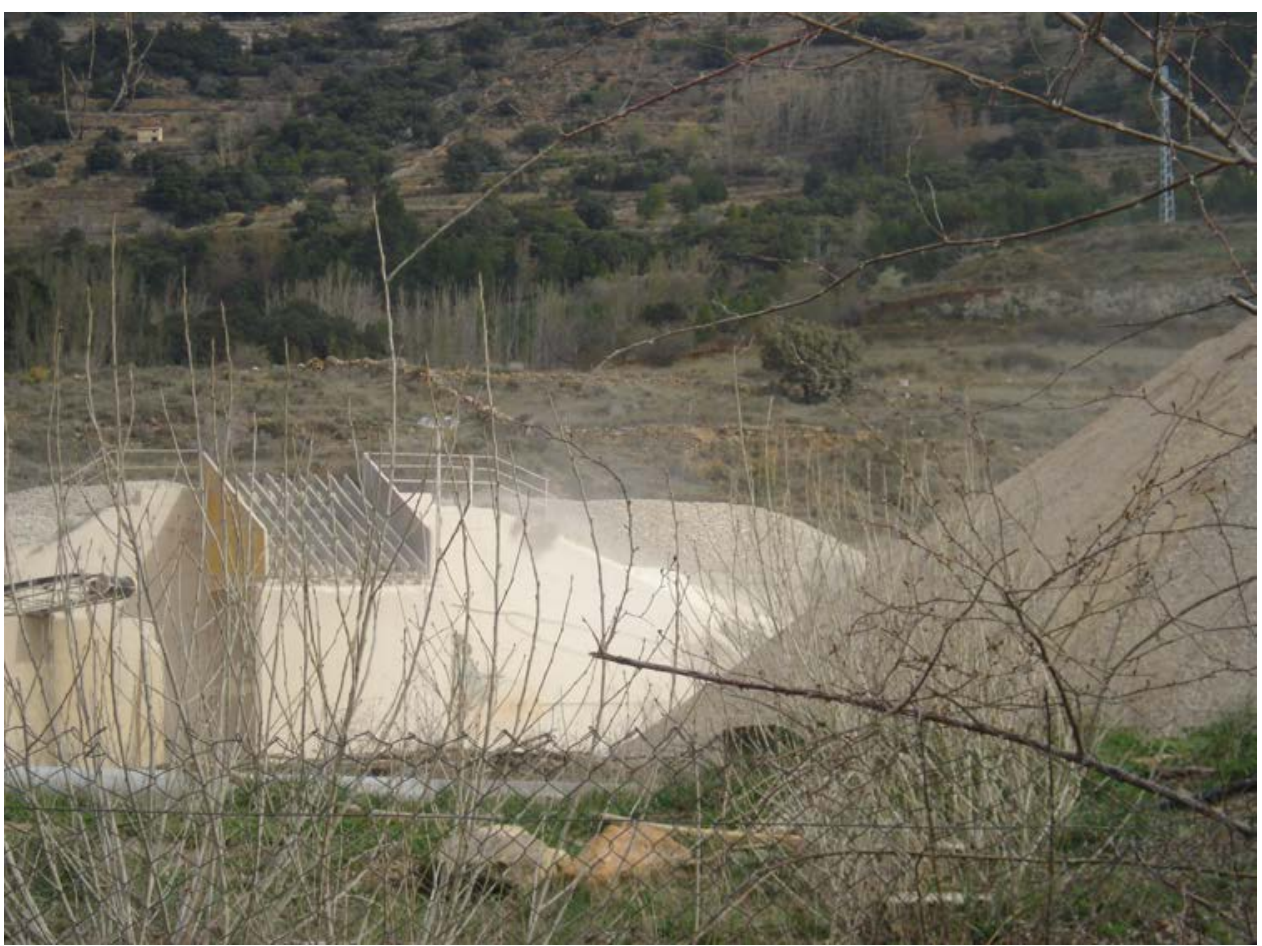

Fotografía 2. Detalle de la gravera del Arroyo de las Truchas. La Iglesuela del Cid

\subsection{Parada 3. EL LOSAR DEL ARROYO DE LAS TRUCHAS, (término municipal de la Iglesuela del Cid, comarca del Maestrazgo). (Hoja 569).}

Después de realizar la parada anterior, cabe retornar a la carretera, con la finalidad de continuar ligeramente hacía el cercano pueblo turolense de la Iglesuela del Cid, (la denominada "Anglesola", por sus vecinos valencianos). Al llegar a la carretera, se hacen ostensibles unas explotaciones de losas. Ahí, a menos de $1 \mathrm{Km}$ de la anterior, haremos otra parada.

En este recorrido, habremos ido encontrando afloramientos de los materiales que ya hemos mencionado en la primera parada. Así, en este lugar afloran unos niveles de calizas arenosas (del Cenomaniense), que son explotados para la obtención de losas para la construcción. Estas losas se ven favorecidas por la estratificación tableada de estas calizas arenosas. (fotografía 3). 


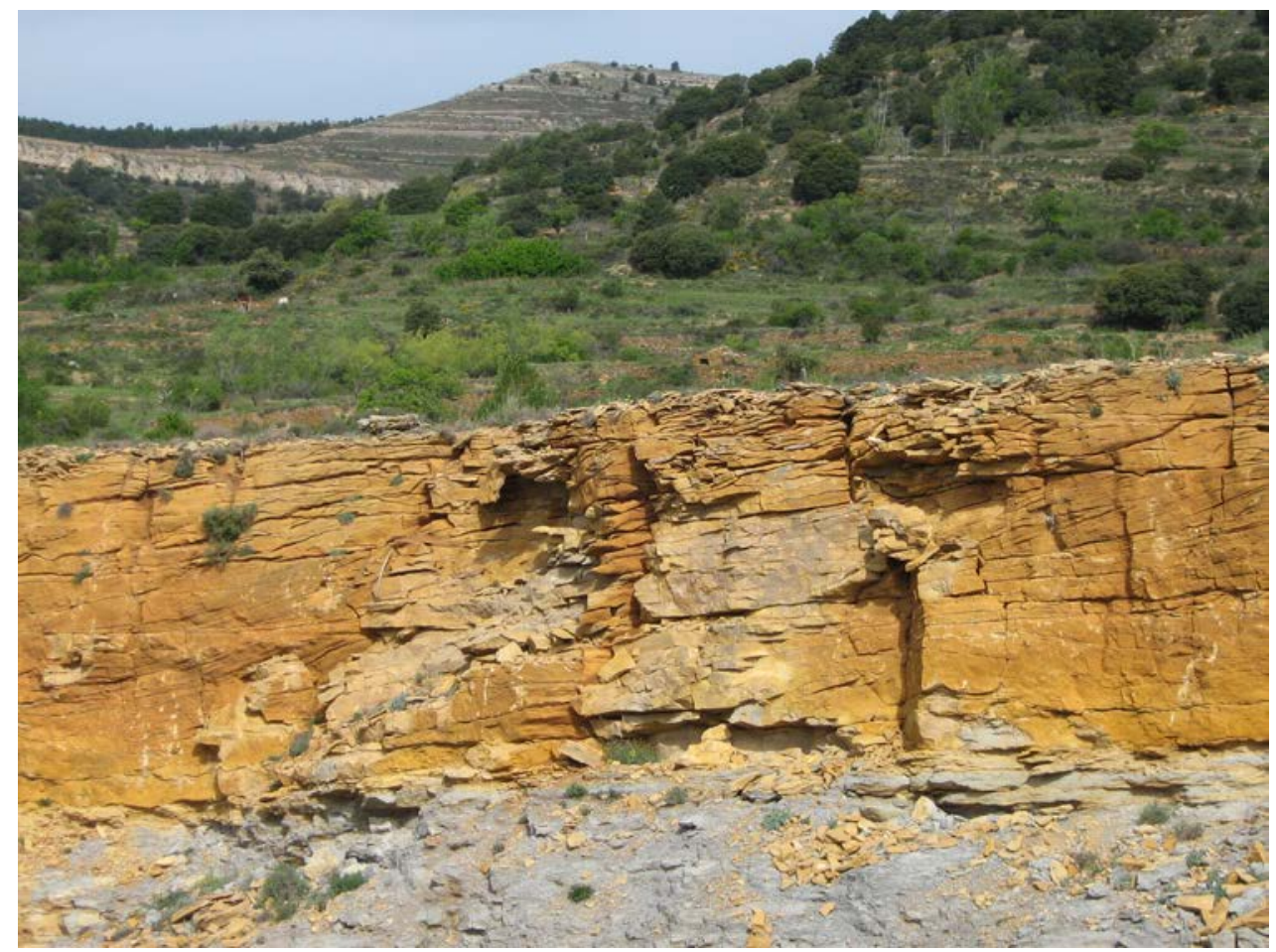

Fotografía 3. Afloramientos de las calizas arenosas en la carretera

\subsection{Parada 4. CARRETERA A MOSQUERUELA. INMEDIACIONES DEL NAVAJUELO, CRUCE DEL SANTUARI DE L'ESTRELLA, (término municipal de Mosqueruela, comarca del Gúdar - Javalambre). (Hoja 569).}

Tras realizar la parada anterior, cabe retornar a Sant Miquel del Ballestar, para después iniciar un recorrido hacia Mosqueruela, utilizando la carretera autonómica valenciana CV - 173. Por ella cabe realizar un recorrido cercano a los $5 \mathrm{Km}$, con la finalidad de llegar hasta el Puerto de la Estrella. En este recorrido, la carretera ha pasado a ser la A - 1701, al tiempo que hemos pasado de la comarca del Alt Maestrat a la de Gúdar Javalambre, de la Comunidad Valenciana a la Comunidad Aragonesa En ese lugar, en las inmediaciones del cerro del Navajuelo, podrá hacerse una nova parada. Así, desde la parada anterior habremos recorrido unos $6 \mathrm{Km}$ más.

En este recorrido habremos encontrado afloramientos de los materiales cretácicos carbonatados. Éstos, en buena parte pertenecen al Cenomaniense y los iremos encontrando en las siguientes paradas, yendo a Mosqueruela. Muy a menudo, habremos visto niveles de calizas arenosas.

En este lugar, mirando hacía el levante, se hace ostensible un sinclinal de dirección claramente catalana, por donde hemos subido. Asimismo, se hacen patentes una serie de pliegues de dirección ibérica y otros de dirección catalana, con claras interferencias entre unos y otros. 


\subsection{Parada 5 - CONDICIONAL. INMEDIACIONES DEL SANTUARIO DE LA VIRGEN DE LA ESTRELLA, (término municipal de Mosqueruela, comarca de Gúdar - Javalambre). (Hoja 569).}

Después de realizar la parada anterior, cabe efectuar un recorrido por la carreterita que se dirige hacia el Santuario de la Virgen de la Estrella, con la finalidad de efectuar una hijuela. Así, tras un recorrido de unos $7 \mathrm{Km}$, llegaremos al mismo.

En este recorrido, hemos ido encontrando afloramientos de los materiales mesozoicos del Cretácico, de los que hemos hablado en los recorridos anteriores. Así, fundamentalmente habremos visto afloramientos carbonatados del Cenomaniense. Estos son los materiales que afloran en el lugar de la parada, en torno al Santuario.

El santuario se halla junto al cauce del Río Montlleó, normalmente seco. Este rio drena parte de los municipios de Mosqueruela, Puertomingalvo y Vistabella del Maestrat. En este lugar va siguiendo un plano de falla, bastante ostensible en el paisaje. (fotografia 4).

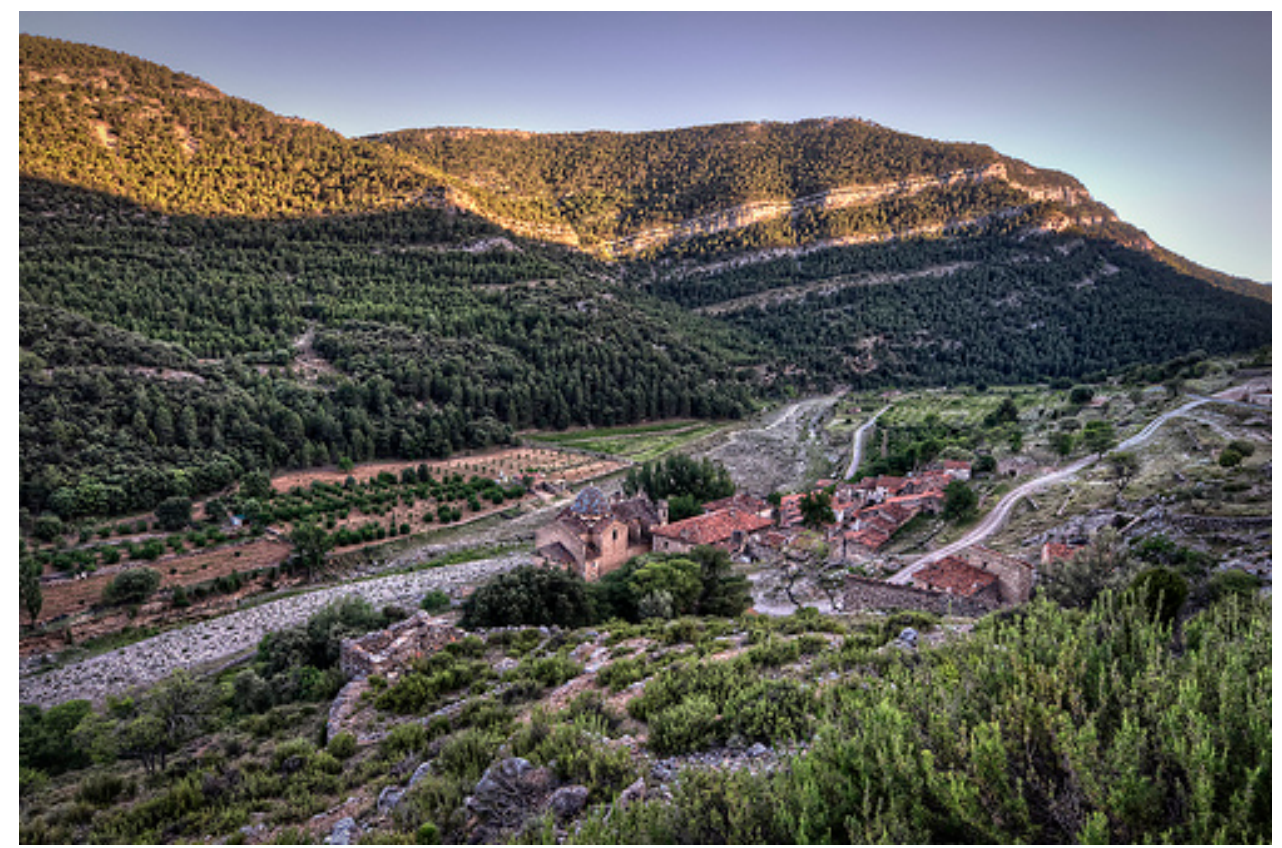

Fotografía 4. El Santuario de la Virgen de la Estrella, junto al río Montlleó. Al otro lado del río, que va siguiendo una fractura, se hacen ostensibles los niveles carbonatados del Turoniense

Fuente imagen: https://www.google.es/search?q=santuario+de+la+estrella+mosqueruela

Por otra parte, los materiales están intensamente fracturados en una zona de interferencias de las direcciones "catalanas" (ENE - WSW) con las direcciones "ibéricas" (WNW - ESE). 


\subsection{Parada 6 - CONDICIONAL. CARRETERA A VILAFRANCA DEL CID, Km 76, (término municipal de Mosqueruela, comarca de Gúdar - Javalambre). (Hoja 569).}

Después de hacer la parada anterior, es necesario retornar a la carretera autonómica aragonesa A - 1701, con la finalidad de continuar hacia el pueblo de Mosqueruela. Sin embargo, a unos $13 \mathrm{Km}$ de la parada anterior ( $\mathrm{y}$ a unos 6 desde el regreso a la carretera), cabe hacer una nueva parada. Ésta la haremos por las inmediaciones del Km 76 (antiguo Km 70) de la carretera.

En este recorrido, hemos ido encontrando afloramientos de los materiales carbonatados cretácicos, de los que hemos hablado en los desplazamientos anteriores. Estos materiales, en su mayor parte pertenecen al Cenomaniense.

Desde este lugar, en donde efectuamos la presente parada, mirando hacia el Sur, puede verse el impresionante barranco de Frares. Este curso de agua es tributario del río Montlleó (que hemos visto en la parada anterior). Así, desde este lugar puede observarse el encajonamiento del barranco y del rio.

\subsection{Parada 7 - CONDICIONAL. CARRETERA A VILAFRANCA DEL CID, Km 69, (término municipal de Mosqueruela, comarca de Gúdar - Javalambre). (Hoja 569).}

Después de realizar la parada anterior, será necesario continuar hacia poniente, por la carretera A - 1701 (acercándonos poco a poco a Mosqueruela). Así, tras efectuar el recorrido de unos $7 \mathrm{Km}$ más, efectuaremos una nueva parada.

Como en los recorridos anteriores, habremos ido encontrando afloramientos de los materiales carbonatados cretácicos. En la mayor parte del recorrido, estos materiales pertenecen al Cenomaniense, como en los recorridos anteriores.

En este lugar, al igual que hemos visto en las paradas anteriores, se puede observar una clara interferencia entre las direcciones ibéricas (WNW - ESE) y las catalanas (NNE - SSW). En efecto, en este lugar pueden verse un sinclinal de dirección catalana, con una serie de pliegues ibéricos que lo interfieren.

\subsection{Parada 8 - CONDICIONAL. CARRETERA A VILAFRANCA DEL CID, Km. 66 (término municipal de Mosqueruela, comarca de Gúdar - Javalambre). (Hoja 569).}

Después de realizar la parada anterior, es necesario continuar el recorrido hacia el Oeste, hacia la cercana población de Mosqueruela. Así, al llegar a las inmediaciones del Km 66, realizaremos una nueva parada, aproximadamente, a unos $3 \mathrm{Km}$ de la realizada anteriormente.

Como en los recorridos anteriores, habremos ido encontrando afloramientos de los materiales carbonatados cretácicos del Cenomaniense, como en este lugar.

En este lugar se hace ostensible una interferencia ibérica - catalana, similar a la que hemos mencionado en las paradas anteriores. Así, mirando hacia el NW, puede verse un anticlinal de dirección ibérica (WNW - ESE), con una serie de pliegues de dirección catalana (NNE - SSW) que lo interfieren. 


\subsection{Parada 9 - CONDICIONAL. POLJÉ DEL BARRANCO DE GILBERT, (término municipal de Mosqueruela, comarca de Gúdar - Javalambre). (Hoja 569).}

Después de realizar la parada anterior, cabe continuar hacía la población de Mosqueruela, siguiendo la carretera A - 1701. Casi al llegar, a unos $3 \mathrm{Km}$ del pueblo y a unos 4'5 Km de la parada anteriormente realizada, cabe la posibilidad de efectuar una nueva parada.

Como en los casos anteriores, en este recorrido, hemos ido encontrando afloramientos de los materiales que hemos mencionado en las paradas anteriores. Así, hemos visto afloramientos de los materiales carbonatados cretácicos, en concreto de los niveles calcáreos (en ocasiones dolomíticos) del Cenomaniense. Estos son también los materiales que aparecen en torno a esta parada.

Por otra parte, en este lugar se hace patente la presencia de poljé, el cual se sitúa a la derecha de la carretera y a la izquierda (yendo hacia el poniente). En este caso, se trata de un pequeño poljé, de dimensiones mucho más reducidas que el poljé de Mosqueruela, que luego veremos. En este caso, cabe hacer constatar que presente poljé tiene un drenaje superficial hacia el rio Malo, afluente del rio Montlleó.

\subsection{Parada 10. EL POLJÉ DE MOSQUERUELA, (término municipal de Mosqueruela, comarca de Gúdar - Javalambre). (Hoja 569).}

Tras realizar el recorrido anterior, cabe continuar por la carretera que se dirige a Mosqueruela la carretera autonómica aragonesa (la A - 1701). Tas sobrepasar el pueblo, efectuaremos una nueva parada, a unos $4 \mathrm{~km}$ de la parada anterior.

En este recorrido, (al igual que hemos efectuado para ir a las paradas anteriores) habremos encontrado afloramientos de los materiales mesozoicos citados anteriormente. Éstos en su mayor parte son de carácter carbonatado (calcáreos), perteneciendo al Cretácico (y fundamentalmente al Cenomaniense).

En este lugar en donde ahora estamos ubicados (a la salida de la población, yendo hacia Linares de Mora o a Puertomingalvo), se patentiza un gran poljé, de dimensiones mayores al de Vilafranca del Cid o al de Vistabella del Maestrat. Pero mucho mayor en este caso, en donde estamos ahora. Para efectuar esta observación, igualmente la podríamos efectuar desde la carretera que conduce Valdelinares.

Así, podemos ver que Mosqueruela se halla situado junto a un inmenso poljé. (fotografías 5 y 6 ). 


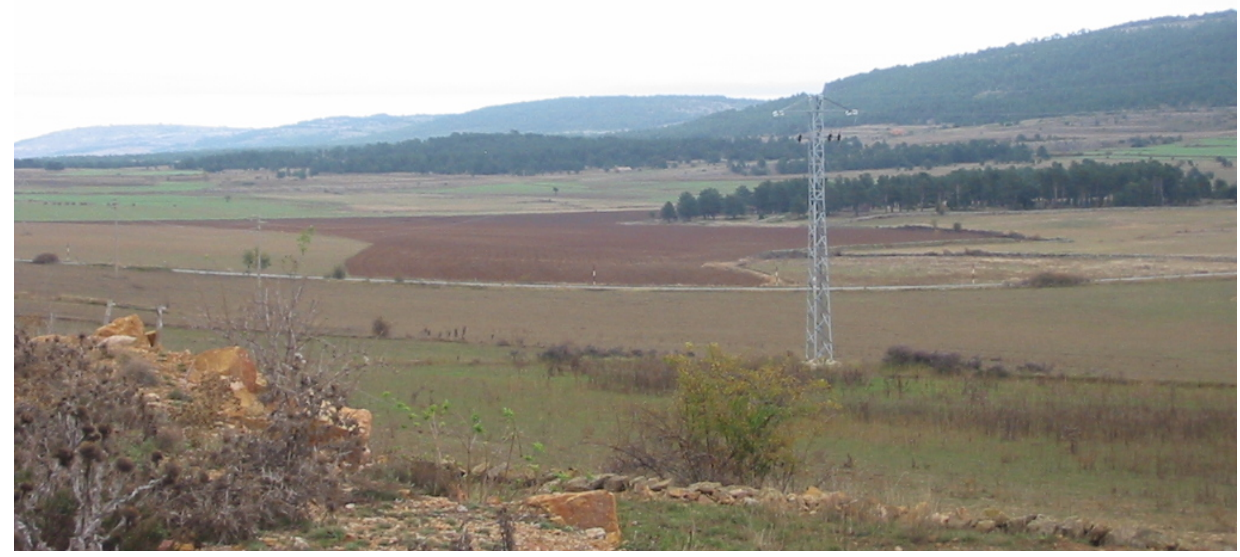

Fotografía 5. Un aspecto del poljé, desde la carretera de Mosqueruela a Puertomingalvo

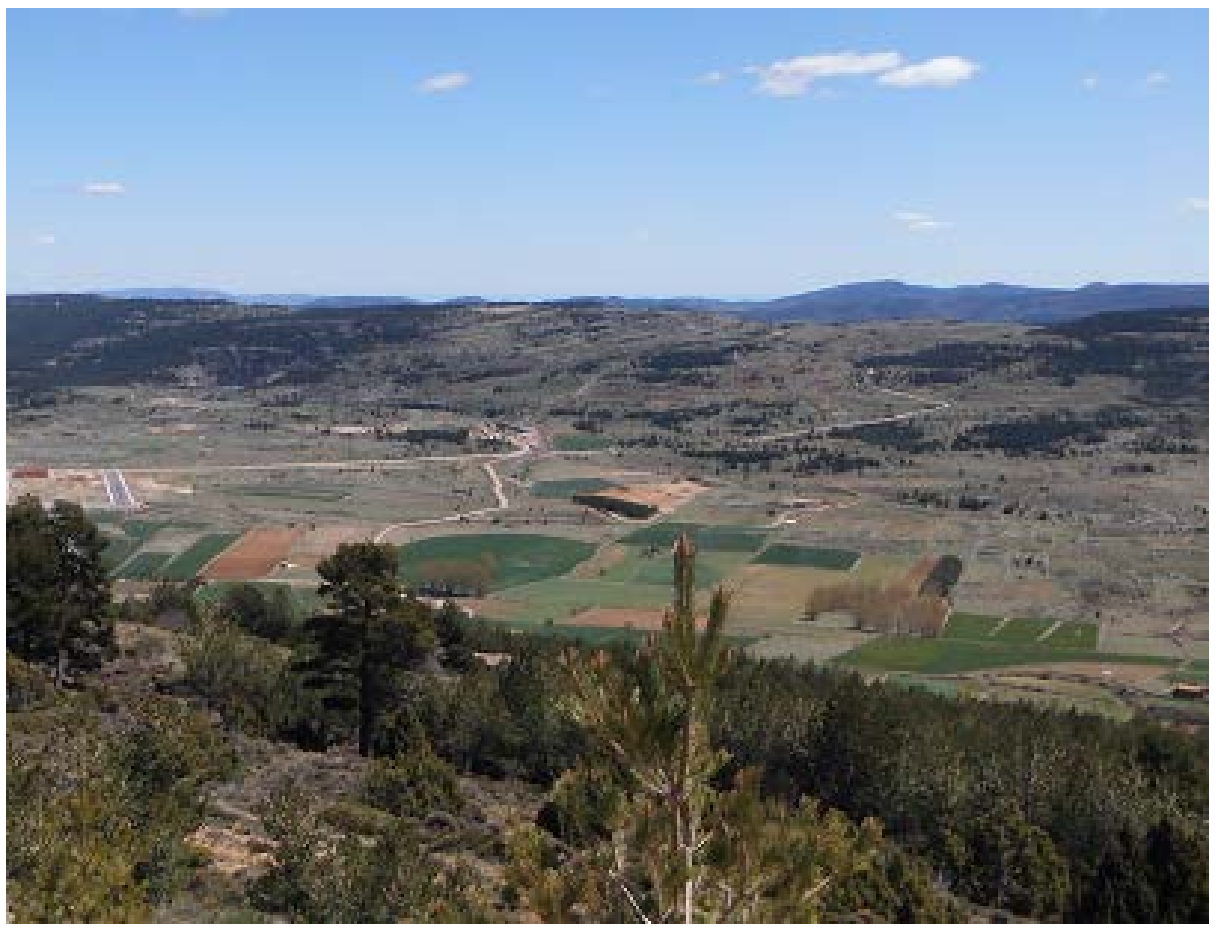

Fotografía 6. El Poljé, des de la carretera de Mosqueruela a Valdelinares Javalambre: desde la Pobla de Sant Miquel al Santuari de l'Estrella y a Mosqueruela 
En este lugar finaliza el recorrido

\subsection{Bibliografía utilizada}

CALVO, M. Et altri (1988).- Minerales de Aragón. Colección: temas geológicos, Edit. Mira, 210 pag. Zaragoza.

GADMA. (2001).- Puntos de Interés Geológico de Aragón, Edit. Gobierno de Aragón, Departamento de Medio Ambiente (GADMA). 243 pag. Zaragoza.

MAESTRE, A. (1845).- Descripción geognóstica del Distrito Minero de Aragón y Cataluña. Anales de Minas, t. III, Madrid.

GUIMERÀ, J. et altri (1992).- Geologia (II), Història Natural dels Països Catalans, Vol. 2, 547 pàg. Enciclopèdia Catalana, S.A.. Barcelona.

IGME (1986).- Mapa Geológico de España a Escala 1:200.000. Explicación de la hoja número: 48 / 5-6 (Vinaròs). Inst. Geol. Min. España. Ministerio de Industria. Madrid.

MATA-PERELLÓ, J.M. (1991).- Inventari de Mineralitzacions i de Minerals del País Valencià. Col-lecció Informe, 546 pag. Manresa.

MATA-PERELLÓ, J.M. (1992a).- Les Mineralitzacions de la Comarca de I'Alt Maestrat, revista Algeps, $\mathrm{n}^{\circ} 1,20$ pàgines, Manresa.

MATA-PERELLÓ, J.M. (1992b).- Síntesi General de les Mineralitzacions de la Regió de Castelló, revista Algeps, nº 7, 35 pàg. Manresa.

MATA-PERELLÓ, J.M. i MONTANÉ GARCIA, P. (2004a).- Recorregut de recerca geològica i mineralògica per les comarques de l'Alt Maestrat, del Maestrazgo i dels Ports: des de Vilafranca del Cid a Cantavieja, Mosqueruela; i de nou a Vilafranca, cap a Castellfort i Morella. Inèdit, 13 pag. Manresa.

MATA-PERELLÓ, J.M. i MONTANÉ GARCÍA, P. (2004b).- Recorregut de recerca geològica i mineralògica per les comarques de la Plana Alta, l'Alcalaten i l'Alt Maestrat: des de la Vall d’Alba i Atzeneta a Vistabella del Maestrat i a Vilafranca del Cid. Inèdit, 13 pag. Manresa.

MATA-PERELLÓ, J.M. i VILALTELLA FARRÀS, J. (2005).- Recorregut de recerca geològica per les comarques del Maestrazgo i de l'alt Maestrat: des de Puertomingalvo a Moqueruela i a Vilafranca del Cid. Inédito. 10 páginas. Manresa.

MATA-PERELLÓ, J.M. i VILALTELLA FARRÀS, J. (2009).- Recorrido geológico y minero por las comarcas del Alcalatén, Gúdar - Javalambre y del Maestrazgo: des de Vilafranca del Cid a Mosqueruela y a la Iglesuela del Cid. Inédito. 10 páginas. Manresa.

PEÑA MONGE, J.L. et altri (1984).- Geomorfología de la provincia de Teruel. Pub. Inst. Estudios Turolenses, 149 pag. Teruel.

RIBA ARDERIU, O. et altri (1976).- Geografia Física dels Països Catalans. Edit. Ketres, 205 pàg. Barcelona. 